\title{
Confinement resonances in photoionization of endohedral atoms: a myth or reality?
}

\author{
A. V. Korol and A. V. Solov'yov \\ Frankfurt Institute for Advanced Studies, Goethe Universität, \\ Ruth-Moufang-Str. 1, 60438 Frankfurt am Main, Germany
}

\begin{abstract}
We demonstrate that the structure of confinement resonances in the photoionization cross section of an endohedral atom is very sensitive to the mean displacement $\langle a\rangle$ of the atom from the cage center. The resonances are strongly suppressed if $2\langle a\rangle$ exceeds the photoelectron half-wavelength. We explain the results of recent experiments which contradict the earlier theoretical predictions on the existence of confinement resonances in particular endohedral systems.

PACS numbers: $32.80 . \mathrm{Fb}, 32.90 .+\mathrm{a}, 36.40 .-\mathrm{c}$
\end{abstract}

In this Letter we explain the discrepancy between theoretical predictions and experimental results for the photoionization (PI) of endohedral atoms $\mathrm{A} @ \mathrm{C}_{N}$. The discrepancy concerns 'confinement resonances' [1] in the PI spectrum whose existence was predicted theoretically [2, ㄱ, 4, 15, 6, 17, 8, 9, 10, 11, 12, 13, 14, 15, 16, 17] (and references therein) but so far has been not supported experimentally [18, 19, 20]. We also formulate the criterion which allows one to estimate, for a particular endohedral system, the interval of photon energies where the resonances can appear.

Confinement resonances appear as a result of interference between a direct wave of the photoelectron escaping the atom and the waves due to scattering from the atoms of the cage. Depending on the photoelectron momentum the interference can be constructive or destructive. Thus, the spectrum of the encaged atom acquires additional oscillations as compared to the free atom. It was noted [2] that the cage-induced oscillations have the same nature as the extended X-ray absorption fine-structure (EXAFS) for solid-state systems.

To date, a number of theoretical investigations have been carried out of the features of confinement resonances for various endohedral systems. The resonances were studied for noble-gas [2, 5, 7, 12, 15] and metal [2, 3, 4, 5, 6, 8, 9, 16] atoms encaged in $\mathrm{C}_{60}$, for $\mathrm{Ar}$ encaged in larger fullerenes $\mathrm{C}_{240}$ and $\mathrm{C}_{540}$ as well as in onion-like structures $\mathrm{C}_{60} @ \mathrm{C}_{240}$ etc 15], for hollow atoms [14]. A strong distortion of the atomic $4 \mathrm{~d}-\mathrm{f}$ giant dipole resonance due to the scattering from the cage was predicted for $\mathrm{Ba} @ \mathrm{C}_{60}$ [2, 3], La@ $\mathrm{C}_{60}$ [2], Xe@ $\mathrm{C}_{60}$ [12] and $\mathrm{Ce}^{3+} @ \mathrm{C}_{60}[16]$. The confinement resonances were also investigated for the angular distribution of photoelectrons [6, 10, 11]. Specific features of the resonances in the PI of endohedral ions $\mathrm{A} @ \mathrm{C}_{60}^{ \pm z}$ were studied in Ref. 13. These and other closely related topics can be found in the recent review [17].

The experimental data on the PI of $\mathrm{A} @ \mathrm{C}_{N}$ are sparse due to '... the difficulty to produce sufficient amounts of purified endohedrals for the gas phase experiments' [20]. So far the PI cross section in the region of the $4 \mathrm{~d}-\mathrm{f}$ giant resonance were measured for $\mathrm{Ce} @ \mathrm{C}_{82}$ [18], $\operatorname{Pr} @ \mathrm{C}_{82}$ [19] and $\mathrm{Ce}_{82} \mathrm{C}_{82}^{+}$20]. In [20] it is explicitly stated that no confinement resonances were detected. With respect to [18, 19] one draws this conclusion matching the measured data to big absolute uncertainties.

Most of the theoretical work has been devoted to the confinement resonances in spherical endohedral systems, in which the atom is placed at the center of a spherical cage. The correlation between the confinement resonances and the off-the-center position of the atom was reported for $\mathrm{Ba} @ \mathrm{C}_{60}$ [4], $\mathrm{Mg} @ \mathrm{C}_{60}$ and $\mathrm{Mg} @ \mathrm{C}_{60}^{ \pm}$[9]. A strong sensitivity of the photoelectron angular distribution and total cross section of PI on the displacement of the atom from cage center was demonstrated [8, 10]. Specific results were presented for $\mathrm{Li} @ \mathrm{C}_{60}$ and $\mathrm{Ar} @ \mathrm{C}_{60}$.

In the cited papers the influence of the off-the-center position was analyzed for the fixed-in-the-space endohedral fullerenes, i.e. fixing both the direction and magnitude of the displacement vector a between the atom and the center. In experimental conditions either one of these parameters or both (depending on the endohedral system) cannot be controlled. As a result, proper averaging procedures must be adopted in order to bring theoretical predictions closer to the observable quantities.

For example, in metallofullerenes $\mathrm{M} @ \mathrm{C}_{N}$ ( $\mathrm{M}$ stands for a metal atom), the atom, as a rule, is located off the center (see, e.g., Ref. 21). The equilibrium can be very stable due to a strong bond of $\mathrm{M}$ to the carbon atoms. Hence, one can fix the distance $a=|\mathbf{a}|$. To explore the properties of an individual metallofullerene the direction of a also can be fixed. However, to compare with the gas phase experiments, any observable, including the cross section, must be averaged over the directions. In this connection we mention Ref. [6] where the atomic 1s PI of $\mathrm{M} @ \mathrm{C}_{60}$ molecules $(\mathrm{M}=\mathrm{Li}, \mathrm{Na}, \mathrm{K})$ was theoretically investigated for the gas of fullerenes. The authors, indicating the off-the-center position of the atom, ignore its influence on the cross section and carry out the study placing the atom at the center for 'computational simplicity' (see the end of Introduction). In what follows we demonstrate, that, generally, such an assumption is not correct and can lead to erroneous predictions on the existence of the confinement resonances. 
Other complexes of current interest are endohedral noble-gas atoms [17]. In these systems the atom stays neutral, and its dynamics is defined by the Lennard-Jones interaction with the cage atoms [22, 23]. The equilibrium position and the amplitude of thermal vibrations depend on the atom type, the cage size and the temperature. For such complexes it is reasonable to carry out also the averaging over the $a$ values.

As we demonstrate below, in many cases the averaging destroys the confinement resonance structure. Prior to introducing the formalism, let us present qualitative arguments. For simplicity, we ignore multiple scattering from the cage. Then, the oscillations in the cross section are due to the interference of the two waves of photoelectron, the direct and the scattered ones, both originating from the same source, - the encaged atom. The atom at the center can be treated as a point-like source. For $\mathbf{a} \neq 0$ the source acquires a size $D \approx 2\langle a\rangle$ where $\langle a\rangle$ is the mean distance from the center. The finite size of the source influences the interference pattern. When $D$ exceeds the half-wavelength the pattern is destroyed. Hence, one can expect that for the photoelectron momenta 24]

$$
p>p_{\min }=\pi / D
$$

the confinement resonances disappear. The corresponding range of photon energies is $\omega>\omega_{\min }=p_{\min }^{2} / 2+I_{0}$, where $I_{0}$ is the ionization potential of the atomic shell.

To start with the formalism let us note that, since the confinement resonances are due to the interference phenomenon, one can adopt the simplest model for electronfullerene interaction which ensures the interference. We model this interaction with a $\delta$-like potential well [5, 25]. Choosing the origin at the atom and introducing the position vector a of the cage center, one writes:

$$
U_{s}(\mathbf{r} ; \mathbf{a})=-A \delta(|\mathbf{r}-\mathbf{a}|-R),
$$

where $A$ can be related to the cage radius $R$ and the electron affinity of the fullerene [5, 25]. For the central position of the atom eq. (2) defines the spherically-symmetric potential $U_{s}(r)=-A \delta(r-R)$ used in the cited papers.

The wavefunction $\Psi_{\mathbf{p}}^{(-)}(\mathbf{r} ; \mathbf{a})$ of the photoelectron emitted from the encaged atom satisfies the equation [26]:

$\Psi_{\mathbf{p}}^{(-)}(\mathbf{r} ; \mathbf{a})=\psi_{\mathbf{p}}^{(-)}(\mathbf{r})-\int \mathrm{d} \mathbf{r}^{\prime} G_{\varepsilon}\left(\mathbf{r}, \mathbf{r}^{\prime}\right) U_{s}\left(\mathbf{r}^{\prime} ; \mathbf{a}\right) \Psi_{\mathbf{p}}^{(-)}\left(\mathbf{r}^{\prime} ; \mathbf{a}\right) .(3)$

The superscript (-) indicates that the asymptotic form is 'plane wave + incoming wave', $\mathbf{p}$ and $\varepsilon=p^{2} / 2$ are the momentum and energy, $\psi_{\mathbf{p}}^{(-)}(\mathbf{r})$ and $G_{\varepsilon}\left(\mathbf{r}, \mathbf{r}^{\prime}\right)$ are the wavefunction and the retarded Green function of the electron escaping from the free atom.

For an at-the-center atom eq. (3) is solved by expand$\operatorname{ing} \Psi_{\mathbf{p}}^{(-)}(\mathbf{r} ; 0), \psi_{\mathbf{p}}^{(-)}(\mathbf{r})$ and $G_{\varepsilon}\left(\mathbf{r}, \mathbf{r}^{\prime}\right)$ in series over spherical harmonics. Then, evaluating the integral and match- ing both sides of the equation at $r=R$, one derives

$$
\begin{aligned}
\Psi_{\mathbf{p}}^{(-)}(\mathbf{r} ; 0)= & \frac{2 \pi}{p r} \sum_{l m} \mathrm{i}^{l} \mathrm{e}^{-\mathrm{i} \delta_{l}}\left(P_{p l}(r)-\frac{\alpha P_{p l}(R) g_{p l}(r, R)}{1+\alpha g_{p l}(R, R)}\right) \\
& \times Y_{l m}\left(\Omega_{\mathbf{r}}\right) Y_{l m}^{*}\left(\Omega_{\mathbf{p}}\right),
\end{aligned}
$$

where $\alpha=2 \pi A$, and $Y_{l m}$ are spherical harmonics. The radial wavefunctions $P_{p l}(r)$ and the scattering phaseshifts $\delta_{l} \equiv \delta_{l}(p)$ correspond to the motion in the field of atomic residue. The radial Green function $g_{p l}\left(r, r^{\prime}\right)$ is proportional to the product of the regular $P_{p l}(r)$ and irregular $\chi_{p l}(r)$ (at $r=0$ ) solutions of the radial Schrödinger equation: $g_{p l}\left(r, r^{\prime}\right)=-P_{p l}\left(r_{<}\right) \chi_{p l}\left(r_{>}\right) / 2 p$ with $r_{>} / r_{<}$being the largest/smallest of $r, r^{\prime}$ (see, e.g., [26]).

Using (4) one relates the amplitude and cross section of PI of the endohedral atom to those of the free atom. For the free atom, the partial amplitude $M_{l l_{0}}^{\mathrm{A}}$ of the dipole transition from the subshell $\nu_{0}=\left(n_{0} l_{0}\right)$ ( $n_{0}$ is the principal quantum number, $l_{0}$ is the orbital momentum) is proportional to the matrix element of the radial dipole operator $\widehat{d}: M_{l l_{0}}^{\mathrm{A}} \propto \int_{0}^{\infty} P_{p l}(r) \widehat{d} P_{\nu_{0}}(r)$ where $P_{\nu_{0}}(r)$ is the shell wavefunction and $l=l_{0} \pm 1$ due to the dipole selection rules. To evaluate the amplitude for the encaged atom one substitutes $P_{p l}(r)$ with the expression in the brackets in (44). Additionally, assuming the shell radius to be much smaller than $R$, one sets $P_{p l}(R) g_{p l}(r, R)=P_{p l}(r) g_{p l}(R, R)$ and derives $M_{l l_{0}}^{\mathrm{A} @ \mathrm{C}_{N}}(\mathbf{a}=0) \approx M_{l l_{0}}^{\mathrm{A}} /\left(1+\alpha g_{p l}(R, R)\right)$. The ratio of the partial cross sections $\sigma_{l l_{0}}^{\mathrm{A} @ \mathrm{C}_{N}}(\omega ; \mathbf{a}) / \sigma_{l l_{0}}^{\mathrm{A}}(\omega) \equiv \eta_{l}(\omega ; \mathbf{a})$, calculated for $\mathbf{a}=0$, reads

$$
\eta_{l}(\omega ; 0)=\left|1+\alpha g_{p l}(R, R)\right|^{-2} \approx 1-2 \alpha \operatorname{Re} g_{p l}(R, R) .
$$

The photon energy $\omega$ is related to $p$ via $p=\sqrt{2\left(\omega+I_{0}\right)}$. The first relation in (5) is similar to the formula derived in [5, 25]. The approximate relation is valid when the cage potential is treated perturbatively.

For momenta $p$ large enough that $p R \gg 1$, one can use the asymptotic formula $g_{p l}(R, R) \propto \exp (-\mathrm{i} p R) \sin (p R-$ $\left.\pi l / 2+\delta_{l}\right)$. Then, eq. (5) explicitly reveals the oscillatory behavior of $\eta_{l}(\omega ; 0)$ as a function of $p$ (or $\omega$ ) due to the interference of the direct and scattered waves.

The exact solution of eq. (3) with the potential (2) taken for the off-the-center position has not been found. However, as mentioned, the cage potential can be treated perturbatively. The first-order solution of (3) reads $\Psi_{\mathbf{p}}^{(-)}(\mathbf{r} ; \mathbf{a}) \approx \psi_{\mathbf{p}}^{(-)}(\mathbf{r})+\Delta \psi_{\mathbf{p}}^{(-)}(\mathbf{r} ; \mathbf{a})$, where

$\Delta \psi_{\mathbf{p}}^{(-)}(\mathbf{r}, \mathbf{a})=A \int \mathrm{d} \mathbf{r}^{\prime} G_{\varepsilon}^{(-)}\left(\mathbf{r}, \mathbf{r}^{\prime}\right) \delta\left(\left|\mathbf{r}^{\prime}-\mathbf{a}\right|-R\right) \psi_{\mathbf{p}}^{(-)}\left(\mathbf{r}^{\prime}\right)(6)$

is the scattered wave. The total amplitude of PI is calculated as $\mathcal{M}^{\mathrm{A}^{\mathrm{C}_{N}}}(\mathbf{a})=\int \mathrm{d} \mathbf{r} \Psi_{\mathbf{p}}^{(-) *}(\mathbf{r} ; \mathbf{a}) \mathbf{e} \cdot \widehat{\mathbf{d}} \psi_{0}(\mathbf{r})=$ $\mathcal{M}^{\mathrm{A}}+\Delta \mathcal{M}(\mathbf{a})$, where $\mathcal{M}^{\mathrm{A}}$ is the amplitude for the free atom and $\Delta \mathcal{M}(\mathbf{a})$ is due to (6). Other notations include 
the unit vector of the photon polarization e, the operator of the dipole moment $\widehat{\mathbf{d}}$, the wavefunction of the subshell $\psi_{0}(\mathbf{r})$. Squaring the modulus of the amplitude and retaining the terms linear in the potential, one writes the cross sections as $\sigma^{\mathrm{A} @ C_{N}}(\omega ; \mathbf{a}) \approx \sigma^{\mathrm{A}}(\omega)+\Delta \sigma(\omega ; \mathbf{a})$, where the term $\Delta \sigma(\omega ; \mathbf{a}) \propto \operatorname{Re}\left(\mathcal{M}^{\mathrm{A}} \Delta \mathcal{M}^{*}(\mathbf{a})\right)$ contains the dependence on a and, thus, must be averaged.

First we consider the angular averaging $\overline{\sigma^{\mathrm{A@C}_{N}}}(\omega ; a) \equiv$ $\int \sigma^{\mathrm{A} @ \mathrm{C}_{N}}(\omega ; \mathbf{a}) \mathrm{d} \Omega_{\mathbf{a}} / 4 \pi$. The dependence on the direction of a enters $\Delta \sigma(\omega ; \mathbf{a})$ via the $\delta$-function from (6). For its averaging one can use the expansion (e.g., 27]): $\delta(|\mathbf{r}-\mathbf{a}|-R)=(2 \pi R / a r) \sum_{l m} P_{l}(\xi) Y_{l m}\left(\Omega_{\mathbf{r}}\right) Y_{l m}^{*}\left(\Omega_{\mathbf{a}}\right)$, where $P_{l}(\xi)$ are the Legendre polynomials, and $\xi=\left(r^{2}+\right.$ $\left.a^{2}-R^{2}\right) / 2 a r$ subject to $|\xi| \leq 1$. Hence $\overline{\delta(|\mathbf{r}-\mathbf{a}|-R)}=$ $R / 2 a r$ if $|\xi| \leq 1$ and $=0$ if otherwise. The rest of the algebra is straightforward. To evaluate the radial integral in $\Delta \mathcal{M}(\mathbf{a})$ one uses the approximation discussed in connection with eq. (5). Finally, noticing that for each partial transition $l_{0} \rightarrow l=l_{0} \pm 1$ the averaged term $\overline{\Delta \sigma}(\omega ; a)$ is proportional to $\sigma_{l l_{0}}^{\mathrm{A}}(\omega)$, one derives the following expressions for the ratio $\bar{\eta}_{l}(\omega ; a)=\overline{\sigma_{l l_{0}}^{\mathrm{A} @ \mathrm{C}_{N}}}(\omega ; a) / \sigma_{l l_{0}}^{\mathrm{A}}(\omega)$ :

$$
\begin{aligned}
\bar{\eta}_{l}(\omega ; a) & =1-\alpha \frac{R}{a} \int_{R-a}^{R+a} \mathrm{~d} r \frac{\operatorname{Re} g_{p l}(r, r)}{r} \\
& =1+(-1)^{l} \alpha\left[S_{p}(a) \cos 2 \delta_{l}+C_{p}(a) \sin 2 \delta_{l}\right]
\end{aligned}
$$

where $S_{p}(a)=(R / 2 p a) \int_{R-a}^{R+a} \mathrm{~d} r r^{-1} \sin (2 p r)$ and $C_{p}(a)=(R / 2 p a) \int_{R-a}^{R+a} \mathrm{~d} r r^{-1} \cos (2 p r)$.

The first relation in (7) qualitatively explains the impact of the angular averaging. For $a=0$ the formula reproduces the right-hand side of eq. (5), derived for the centrally positioned atom treated as a point-like source. As $a$ increases the averaging leads to a non-zero effective size of the source, $D=2 a$. When $D$ becomes larger than the emitted half-wavelength the interference is lost. Hence, for $p>p_{\min }=\pi / 2 a$ the confinement resonances in the cross section profile disappear.

The right-hand side of (17) allows one to analyze quantitatively the modification of the interference pattern without calculating the parameters of photoelectron wavefunction (in particular, the phaseshifts) but using only $p$, $R$ and $a$. Indeed, the relative change in the amplitude of the oscillations can be understood by comparing the functions $S_{p}(a)$ and $C_{p}(a)$ to their values at $a=0$.

Let us apply eq. (77) to estimate the effect for $\mathrm{Ce}_{0} \mathrm{C}_{82}$. In [18, 20] the PI cross section was measured in the region of the $4 \mathrm{~d}$ giant dipole resonance, i.e. for $\omega=120 \ldots 140$ $\mathrm{eV}$, and no oscillations were seen. The $4 \mathrm{~d}$ ionization potential in Ce is $114 \mathrm{eV}$ for $4 \mathrm{~d}_{3 / 2}$ shell and $111 \mathrm{eV}$ for $4 \mathrm{~d}_{5 / 2}$ shell 28]. Hence, the indicated range of $\omega$ corresponds the photoelectron momenta $p=0.5 \ldots 1.5$ a.u. The mean radius of $\mathrm{C}_{82}$ is $4.15 \AA$ and $\mathrm{Ce}$ atom is displaced by $1.8 \AA$ from the center 29]. The dependences $S_{p}(a)$ and $C_{p}(a)$ on $p$ are presented in Fig. 1. It is clearly seen that angular averaging destroys the interference for $p>p_{\min } \approx 0.46$ a.u, i.e. for the photon energies $\omega \gtrsim I_{0}+3 \mathrm{eV}$.

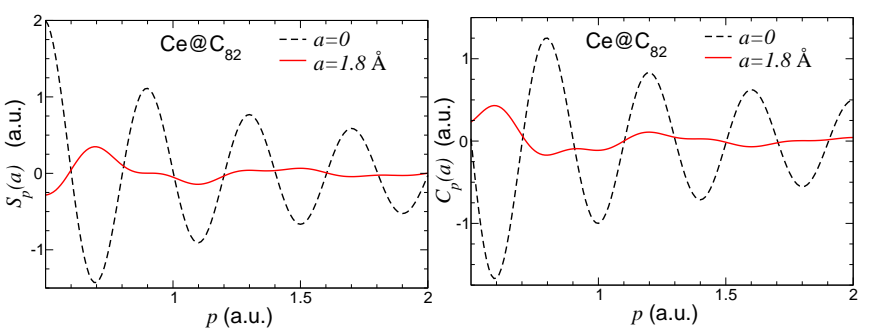

FIG. 1: $S_{p}(a)$ and $C_{p}(a)$ versus $p$ calculated for Ce@ $\mathrm{C}_{82}$ for at-the-center position of Ce and off-the-center with $a=1.8 \AA$.

Similar estimates explain the absence of the confine-

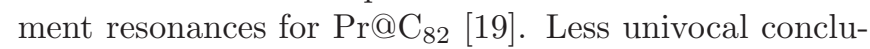
sion can be drawn for $\mathrm{Li} @ \mathrm{C}_{60}, \mathrm{~K} @ \mathrm{C}_{60}$ and $\mathrm{Mg} @ \mathrm{C}_{60}$ the PI of which was considered theoretically [6]. The authors indicate that the atoms displacements are 1.5, 0.25 and $1 \AA$ for $\mathrm{Li}, \mathrm{K}$, and $\mathrm{Mg}$, respectively, but set $a=0$ when calculating the cross section. As a result, for each metallofullerene they predicted several confinement resonances for the photoelectron energies below 2 a.u. However, calculating $\varepsilon_{\min }=p_{\min }^{2} / 2$ and obtaining the values $0.15,5.5$ and 0.35 a.u., we state that the resonances in $\mathrm{Li} @ \mathrm{C}_{60}$ and $\mathrm{Mg} @ \mathrm{C}_{60}$ will hardly survive the averaging procedure, whereas the resonances predicted for $\mathrm{K}_{0} \mathrm{C}_{60}$ will be preserved due to small value of $a$.

To develop the theory further one accounts for thermal vibrations of the atom in the vicinity of equilibrium position. This can be important, e.g., for endohedral noble-gas atoms. Let us construct a model potential for the $\mathrm{A}-\mathrm{C}_{N}$ interaction which, despite being quite crude, allows one to analyze the influence of the vibrations on the interference pattern. Ignoring the atom-fullerene hybridization one builds the potential as a sum of pairwise A-C Lennard-Jones potentials over all carbon atoms [22]. Assuming a homogeneous distribution of carbon atoms over the sphere of radius $R$ and substituting the sum with the surface integral, one derives the $\mathrm{A}-\mathrm{C}_{N}$ potential $U(a)=U_{\mathrm{L}}(a)+U_{\text {ex }}(a)$ as a function of $a[30$. The term $U_{\mathrm{L}}(a)$ is the attractive (the London-type) potential, and $U_{\text {ex }}(a)$ is the repulsive exchange term.
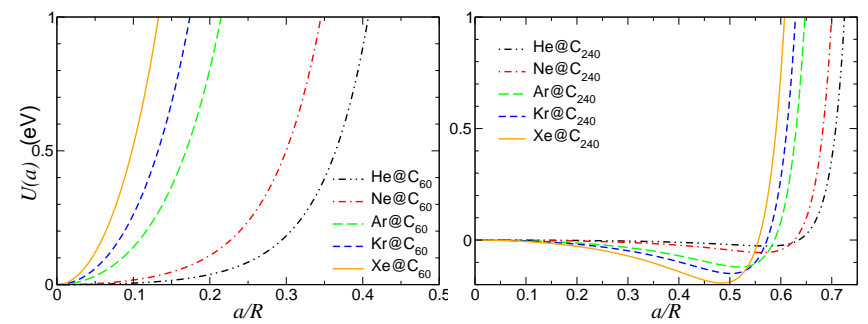

FIG. 2: Potentials $U(a)$ for various $\mathrm{A} @ \mathrm{C}_{N}$ as indicated.

The functions $U(a)$ for various systems are presented in Fig. 2. The radius $3.5 \AA$ of the $\mathrm{C}_{60}$ cage is less (for $\mathrm{Ar}$, 
$\mathrm{Kr}, \mathrm{Xe})$ or just above (He, Ne) the equilibrium distance of the Lennard-Jones potential [22], so that the atom is confined to the center 31]. For $\mathrm{C}_{240}$ the radius 7.1 $\AA$ exceeds the equilibrium distance. Hence, the atom is either weakly bound well off the center (Ar, $\mathrm{Kr}, \mathrm{Xe}$ ) or moves freely in most part of the fullerene $(\mathrm{He}, \mathrm{Ne})$.

The probability to find an atom at the distance $a$ is given by $\mathrm{d} W_{T}(a)=C \exp (-U(a) / k T) a^{2} \mathrm{~d} a$, where $T$ is the temperature, $k$ - the Boltzmann constant, and $C=$ $\left[\int_{0}^{a_{\max }} \mathrm{d} W_{T}(a)\right]^{-1}$ with $a_{\max } \approx R-R_{\mathrm{A}}\left(R_{\mathrm{A}}\right.$ is the radius of the atom). To average the ratio $\bar{\eta}_{l}(\omega ; a)$ over $a$ one multiplies eq. (7) by $\mathrm{d}_{T}(a)$ and integrates over $a$ :

$$
\left\langle\bar{\eta}_{l}(\omega)\right\rangle=1+\alpha\left[\left\langle S_{p}\right\rangle \cos 2 \delta_{l}+\left\langle C_{p}\right\rangle \sin 2 \delta_{l}\right]
$$

with the general notation $\langle g\rangle=\int_{0}^{a_{\max }} g(a) \mathrm{d} W_{T}(a)$.

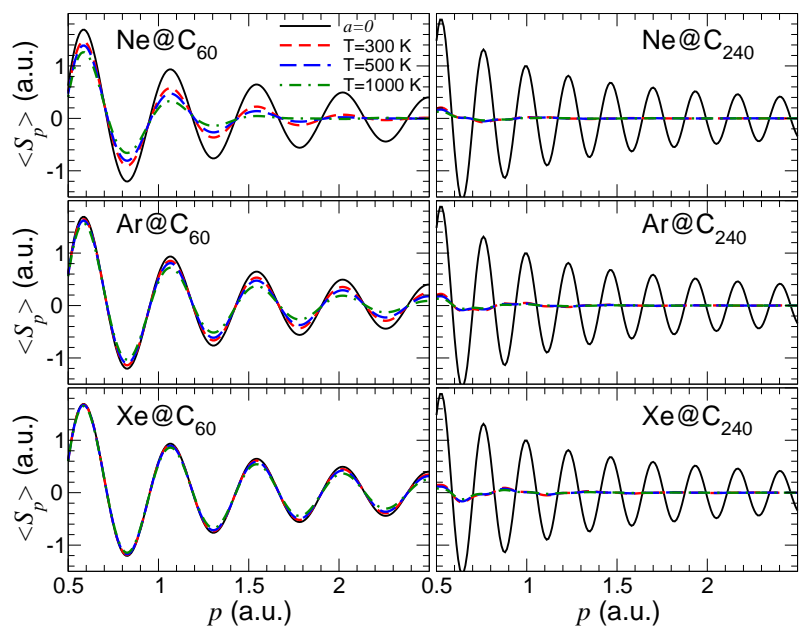

FIG. 3: $\left\langle S_{p}\right\rangle$ versus $p$ calculated for various $T$ and $\mathrm{A} @ \mathrm{C}_{N}$.

Dependences $\left\langle S_{p}\right\rangle$ on $p$ are presented in Fig. 3 [32. It is seen that in all $\mathrm{A} @ \mathrm{C}_{240}$ systems the oscillations are suppressed because of strong off-the-center position of the atom. The situation is not so universal for a smaller $\mathrm{C}_{60}$ fullerene in which the equilibrium is at the center. Indeed, for Ne the amplitude of oscillations decreases noticeably for all $T$ starting with $p \approx 1$ a.u., whereas for Xe the oscillations stay nearly unchanged up to $p \gg 1$ a.u. To explain the difference one estimates the effective size of the source as the doubled mean amplitude of thermal vibrations and calculates the momenta $p_{\min }=\pi / D$ beyond which the interference disappears. For $T=500$ $\mathrm{K}$ the calculated values of $p_{\min }$ are 1.8, 3.9, 7.1 a.u. for $\mathrm{Ne} @ \mathrm{C}_{60}, \mathrm{Ar} @ \mathrm{C}_{60}$ and $\mathrm{Xe}_{0} \mathrm{C}_{60}$, respectively.

In summary, we have demonstrated that the interference effects in photoionization of $\mathrm{A} @ \mathrm{C}_{N}$ are very sensitive to the mean displacement $\langle a\rangle$ of the atom from the cage center. The range of photon energies, where the confinement resonances can be observed, one deduces by matching $2\langle a\rangle$ the photoelectron half-wavelength. In metallofullerenes, due to a non-central position of the atom, the angular averaging can destroy the oscillatory structure predicted for the atom at-the-center. For large noble-gas endohedral fullerenes $\left(\mathrm{C}_{240}\right.$ and larger $)$ the resonances do not survive the averaging procedure with the Boltzmann distribution function. For a smaller $\mathrm{C}_{60}$ fullerene a more rigorous treatment of the atom-fullerene interaction is needed to draw the final conclusion on the existence of the resonances. The role of non-centrality must be studies for other related phenomena (Coulomb confinement resonances, non-dipole effects, the photoionization of onion-like structures etc [17]).

The work was supported by the European Commission within the NoE project EXCELL (Project No 515703).

[1] J.-P. Connerade, V.K. Dolmatov, and S.T. Manson, J. Phys. B 33, 2279 (2000).

[2] M.J. Puska and R.M. Nieminen, Phys. Rev. A 47, 1181 (1993); 49629 (1994) (Errata).

[3] G. Wendin and B. Wästberg, Phys. Rev. B 48, 14764 (1993).

[4] J. Luberek and G. Wendin, Chem. Phys. Lett. 248, 147 (1996).

[5] A.S. Baltenkov, J. Phys. B 32, 2745 (1999).

[6] P. Decleva et al., J. Phys. B 32, 4523 (1999).

[7] M.Ya. Amusia, A.S. Baltenkov, and U. Becker, Phys. Rev. A 62, 012701 (2000).

[8] A.S. Baltenkov, V.K. Dolmatov, and S.T. Manson, Phys. Rev. A 66, 023201 (2002).

[9] M. Aichinger et al., J. Mod. Opt. 50, 2691 (2003).

[10] A.S. Baltenkov et al, Phys. Rev. A 68, 043202 (2003).

[11] M.Ya. Amusia et al, Phys. Rev. A 70023201 (2004).

[12] M.Ya. Amusia et al., J. Phys. B 38, L169 (2005).

[13] V.K. Dolmatov and S.T. Manson, Phys. Rev. A 73 013201 (2006).

[14] A.S. Baltenkov, S.T. Manson, and A.Z. Msezane, Phys. Rev. A 76, 042707 (2007).

[15] V.K. Dolmatov, P. Brewer, and S.T. Manson, Phys. Rev. A 78013415 (2008).

[16] Z. Chen and A.Z. Msezane, J. Phys. B 42, 165206 (2009).

[17] V.K. Dolmatov, in Theory of Confined Quantum Sytems: Part 2, edited by J.R. Sabin and E. Brändas, Adv. Quant. Chem. (Academic Press, New York, 2009), Vol. 58, p. 13.

[18] K. Mitsuke et al., J. Chem. Phys. 122, 064304 (2005).

[19] H. Katayanagi et al., J. Quant. Spectrosc. Radiat. Transfer 109, 1590 (2008).

[20] A. Müller et al., J. Phys.: Conf. Ser. 88012038 (2007).

[21] H. Shinohara. Rep. Prog. Phys. 63, 843 (2000).

[22] V.V. Albert et al., Int. J. Quant. Chem. 107, 3061 (2007).

[23] P. Pyykkö et al., Phys.Chem.Chem.Phys. 9, 2954 (2007).

[24] The atomic system of units is used in the paper.

[25] M.Ya. Amusia, A.S. Baltenkov, and B.G. Krakov, Phys. Lett. A 243, 99 (1998).

[26] A. Messiah, Quantum mechanics (North-Holland, Amsterdam, 1999).

[27] D.A. Varshalovich, A.N. Moskalev, and V.K. Khersonskii, Quantum Theory of Angular Momentum (World Scientific, Singapore, 1988).

[28] A.A. Radzig and B.M. Smirnov, Reference Data on Atoms, Molecules and Ions (Springer, Berlin, 1985).

[29] S. Nagase et al., Chem. Phys. Lett. 201, 475 (1993); 
C.J. Nuttall et al., Mol.Cryst. Liq.Cryst. 340, 635 (2000).

[30] S. Lo, A.V. Korol, and A.V. Solov'yov, Phys. Rev. A 79 063201 (2009).

[31] The model ignores the plasmon excitations in $\mathrm{C}_{N}$, which enhance $U_{\mathrm{L}}(a)$ 23] and make the curve $U(a)$ less steep. [32] General trends of $\left\langle C_{p}\right\rangle$ are similar. To save space we do not include the corresponding graphs. 